\title{
Relational Ethics and Psychosomatic Assessment
}

\section{António Barbosa}

Center for Bioethics, Faculty of Medicine, University of Lisbon, and Psychiatry Department, Hospital de Santa Maria, Lisbon, Portugal

\begin{abstract}
The main ethical perspective in the clinical relationship takes into consideration the vulnerability of the clinical condition before threats and risks that can undermine the integrity and dignity of the person. Psychosomatic medicine faces complex cases whose ethical problems cannot only be solved by applying top-down deontological or utilitarian approaches, principlism, which is limited mainly to easing ethical tensions, or a bottom-up approach, the casuistic model, case-based reasoning. In introducing vulnerability as the core of ethical questioning as a principle ontological priority over other principles, relational ethics refers to the appreciation of the responsibility of health professionals through which a health care professional and the patient 'together' can construct more reasonable and prudential courses of action with, for, and by the patient. The model of relational ethics is based on three main aspects, clinically integrated approach, science/philosophy partnership, and deliberative process, that when taken together, form an intermediate model that ensures prudent and reasonable decision-making. The three structural elements and characteristics of relational ethics create and maintain a responsible relationship between the professional and the patient being aware that the mutual vulnerability of health professional and the patient has a moral value and recognizing that their relationship will allow for personal development of each. I conceptualized the model of relational ethics as one that embraces the meta-ethical principles of vulnerability, dignity, responsibility, and respect for autonomy as they are considered by many international declarations or conventions. This model integrates three key polarities: ensure conditions of authenticity, facilitate a process of cooperative mutuality, and promote opportunities for growth and development. Relational ethics can be used to solve major ethical problems in psychosomatic medicine, capacity, informed consent, and confidentiality.

Copyright $\odot 2012$ S. Karger AG, Basel
\end{abstract}

\section{Ethical Foundations}

Bioethics in its essence is an experience of duty or obligation that arises from an intelligent facing of events, given a virtually unlimited range of possibilities against which 
one has to freely do his own adjustment. That is, one's actions must be justified. This justified or facere-iustum ('to make just') basis of actions is precisely the moral life [1].

Otherwise, bioethics should be understood as a category in itself, without trying to reduce it to any of its components. Contributions from biology, psychology, psychiatry, psychosomatics, and other disciplines provide necessary but not sufficient conditions for ethical conduct. Ethical conduct is irreducible to any other conduct. There is a pathology of morality, but that does not mean that morality is always pathological.

In the field of ethics, therefore, it is necessary to distinguish between the specific contributions of psychiatry and of psychosomatic medicine. Sometimes, these contributions are interrelated, as in, for example, the following circumstances in which a health professional (1) believes that all conflicts of values (arising, for example, when a patient has different values from professional values and feels compelled to assert them in his relationship with the health professional) come from a mental disorder such as disease phobia; (2) systematically puts the patient under suspicion of having a psychological or mental problem and is unable to decide, as in illness denial, and (3) considers that ethical problems are misplaced technical problems or unresolved psychological or mental conflicts, such as demoralization.

In short, we are led to regard ethics as pathologic and its management as to be treated psychiatrically, which constitutes an unacceptable reductionism. This reductionism is often merely the result of a health professional's anxiety, lack of knowledge of ethics and even of psychology.

Reflecting on the ethical problems in the practice of psychosomatic medicine will improve the patient's functioning, improve the care of his family and community, and lead to a better assessing of the patient's function. Such reflection will increase the understanding that the psychosomatic patient must be respected in his autonomy despite his having malfunctions or disturbances.

Some ethicists continue to consider the psychiatric substrate irrelevant to a discussion of values (as in the genetic fallacy). Others, however, recognize the important role of psychological factors in cognition. They find that the role of psychological factors to be useful because it enriches the dialogue between ethics and psychosomatics.

Psychosomatic medicine has specialized in understanding not only the rational elements of ethical reasoning but also the irrational elements, and also the psychological, developmental, and environmental factors determining the elements of ethical reasoning. Ethical conflicts commonly arise in the context of emotional stress, such as intolerable anxiety and confusion, and in the context of conflict. With their allostatic load, ethical conflicts can make some patients, families and even the teams exacerbate dysfunctional preexisting problems. This distress can lead to malfunctions and even also to psychiatric disorders. Although these consequences of distress do not prevent the process of decision-making, they do inject confusion and distortion. These situations require specific assessments with new ethical methods.

Psychosomatic medicine faces complex cases entailing the following: (1) multisystem evaluation both in type and number of organ systems involved, and 
interconnection or interdependence between the systems involved and the biological, psychological, and social impact in the disease; (2) multiple therapies that are not always well defined and articulated in a plan of care with mutual responsibility between patient and professional, and (3) a multidisciplinary approach involving health professionals trained in an atmosphere of rigid discipline and overspecialization that controls the extents of their knowledge and practice and that uses specific theories and terminologies.

These characteristics of patients and health systems create technical-professional problems but also, increasingly, ethical problems that cannot be entirely solved by the mechanical application of top-down models such as the determination of a deontological or a utilitarian model.

In the deontologic model, it is difficult to apply a single moral principle with reference to a particular difficult and complex clinical case given. The reason is that the deontologic model rejects the idea of paying attention to anthropological and situational assessment of the circumstances of the case. It is difficult to apply the Kantian categorical imperative to particular cases without an intermediate approach. Kantian ethics is especially powerful for the application of a second formulation of the categorical imperative (humanity as an end in itself) that serves to protect patients' rights against forms of exploitation of human beings.

Moreover, despite that the consequentialist-teleologic model and utilitarian approaches pay more attention to the anthropological data and the assessment of the circumstances, they have some constraints on the two following aspects: (1) in a strict limitation of the consequences of particular actions, it is not always appropriate to leave out the intentions of the actors, and (2) consequences such as 'the greatest good for the greatest number' may endanger respect for the problems of minority populations.

One of the rules of ethics applied to health practices forms what became known as 'principlism'. The principles outlined in the Belmont report served as a guide to many of the ethical discussions of the 1970s and 1980s [2]. Markedly influenced by the use of technology in medicine, such as in treatment options, principlism is limited mainly to the mere resolution of ethical tension among the rights of the patient (respect for autonomy), the virtue of the professionals (beneficence and nonmaleficence), and social responsibility of fairness (justice). However, in the reality of clinical practice, these principles are often synergistic and do not explicitly incorporate the principles of vulnerability and responsibility, which are fundamental dimensions of the clinical relationship.

Some of the disadvantages of principlism are offset by a bottom-up model such as the casuistic model, case-based reasoning. The casuistic model is more sensitive to particular aspects of particular cases than is the application of abstract universal norms or of general rules to paradigmatic cases. By being based directly on particular cases, the casuistic model has the great advantage of practical relevance. Furthermore, reasoning about cases is something that all practitioners are familiar with in other clinical contexts, and casuistic reasoning can often lead to agreement. 
However, the casuistic model raises some problems, starting with the definition of what a paradigmatic case is.

Unless a health professional has some references to universal principles, the casuistic model risks falling into relativism by focusing on the expectations and values that are culturally and communally determined. Another problem with the casuistic model is that it can be overly loose and unstructured.

And yet a more significant difficulty of the casuistic model, especially considering the importance of different value perspectives in mental health, is its danger of reinforcing prejudices. Casuistic reasoning arrives at agreement only when the perspectives and values of those concerned are shared. This is not always the case [3].

Given these limitations in the casuistic model, it is desirable that in ethical decisionmaking, justified reasons can be made. This means that the arguments can call up some universal agreement leaving the conviction that the decision is not fully processed by contingent facts as dependent on certain contexts. It must assume some form of 'universality', i.e. make the decision available to all persons connected with the same situation.

Casuistry was developed in part as a response to the misuse of principlism, and it is more compatible with clinical medical practice and narrative ethics than is principlism. Casuistry regards experiential and interpersonal aspects such as vital issues in clinical practice as it also occurs with the recent development of care ethics, hermeneutics, and responsibility ethics (fig. 1).

The clinical relationship is created in a space and time that are necessary conditions for the emergence of patients' and professionals' experiential values through which they can build a relationship of trust. It is from observing that the clinical relationship is not merely a procedural application of principles, such as obtaining formal informed consent, through which other ethical perspectives have emerged.

The main ethical perspective in the clinical relationship is taking into consideration the vulnerability of the clinical condition before threats and risks that can undermine the integrity and dignity of the person. These aspects are deepened by a responsibility ethics (principles of precaution/security) and with care/narrative/hermeneutic ethics (principle of solicitude). The vulnerability of the patient is also approached by the principles of protection, solidarity, and nondiscrimination. In the relationship between society with individuals and families, some important regulatory processes can be ethically processed by the rules of discussion ethics.

\section{Relational Ethics}

The key vector of the new outlook is a shift from focusing on reifying patient autonomy to focusing precisely on the problems of limitation of autonomy that are related to loss and to risks and to threats to the integrity and dignity of human beings. This refocus introduces such vulnerability as the core of ethical questioning, as a principle ontological priority over other principles [1]. 


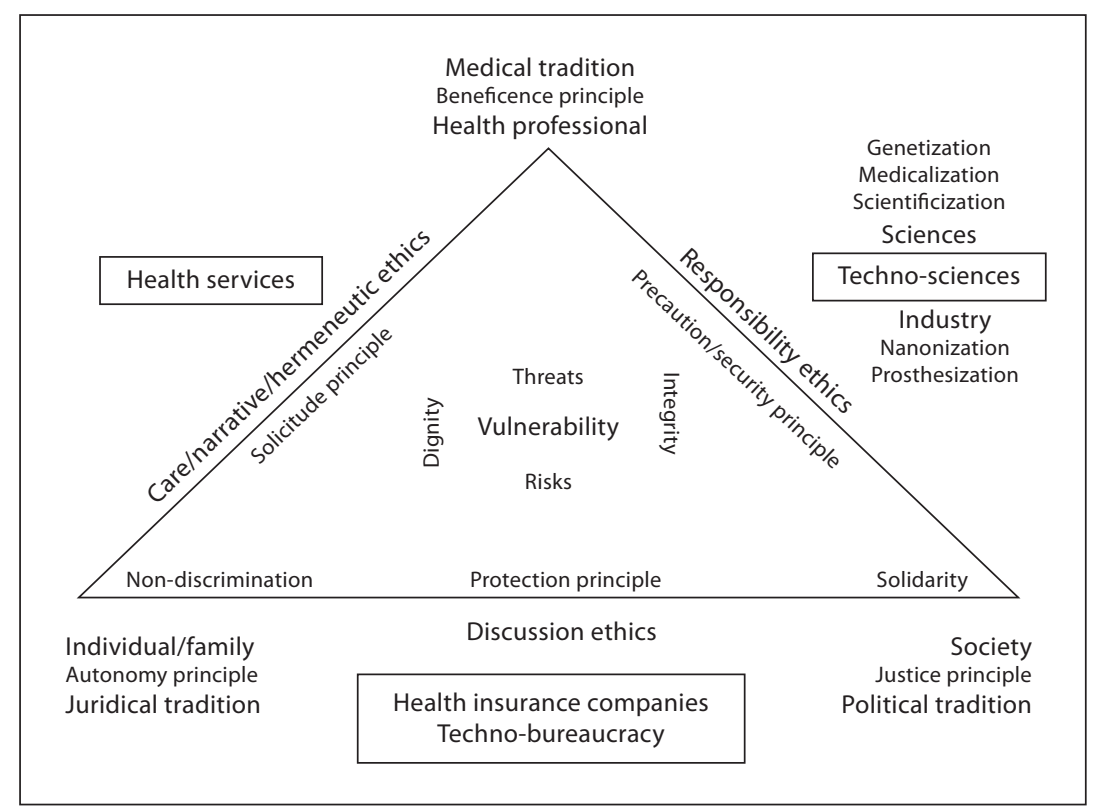

Fig. 1. Ethical principles: theoretical framework.

In the clinical relationship, the interactive process between the health professional and the patient often begins by the awakening of the ethical core (ethical concern). This core allows a climate of mutuality and creative cooperation that underpins the formulation of a commitment and a promise of help in a sequenced scientific-technical and human action. Within a deontologic context of good practice, technical means appropriate to clinical situations are mobilized. This process begins in creating conditions for developing confidence in the relationship between the health professional and the patient. It is through this process (which comprises authenticity, cooperative mutuality, and the opportunity for personal development) that the health professional and the patient can, within a fixed set of principles, share interpretations of situations such as current experiences and their historical and contextual backgrounds, while at the same time affording openness and flexibility to accommodate new, emerging possibilities.

By referring to different theoretical frameworks, we can better apply relevant ethical issues in analyzing a situation. However, it is also necessary to give importance to relational responsibility.

In the ethical practice of psychosomatic medicine, relational ethics refers to the appreciation of the responsibility of health professionals. This appreciation is not only to terms of fidelity to duty and obligation of respect for the person or to submission to general rules, such as 'the best for the majority', but also to the development of a relational commitment between the caregiver and one for whom he or she is responsible. 
It is through this middle range theory, which I refer to as relational ethics, which a health care professional can define the ethical conditions that through a relational process between the health care professional and the patient, each can come into contact with the authentic reality of the other. In this way, a health care professional and the patient 'together' can construct more reasonable and prudential courses of action with, for, and by the patient. It is therefore necessary to establish the possible conditions that allow an integrated deliberative process.

Meta-analytically, I refer my relational model of ethics to an ethics of dialogue, narrative, hermeneutics, and pragmatics that requires openness to the perspectives of others to the contingency, to the context, and to the specific/pragmatic situation. The importance of the practical processes of the production of meaning is stressed.

This model distinguishes itself from other models, such as principlism, in which ethical problems are often discussed strictly in terms of defined principles, are formulated through broad ahistorical interpretations, and are resolved through abstract procedures.

This model of relational ethics is based on three main aspects: clinically integrated approach, science/philosophy partnership, and deliberative process.

Clinically integrated approach:

1 The problems in health are complex but simultaneously concrete (practical rationality). The ethical dimension is fulfilled only in a specific situation (pragmatic) that takes shape only through clarification dialogue/narrative of this particular ethos [4], and understood by a hermeneutical perspective because understanding is always interpret and to interpret is to apply.

2 An approach centered on a case is more consistent with the way health professionals approach diagnosis and treatment than is an emphasis on rules, principles, and generalizations. Such an emphasis obscures the importance of an individual case and its concrete situation.

3 A focus on each stage of the clinical process, particularly on issues of treatment choice and ethical reasoning, is fully integrated with other clinical practice skills. A focus on traditional bioethics lacks this integration.

Science/philosophy partnership:

1 The relationship between ethics and science is mainly regarded as one of partnership rather than of moral guardianship.

2 Work in the philosophy of science, in this instance on epistemic values in psychiatric classification, becomes partnered with the philosophy of mind, such as on rationality, meaning, agency, and identity [5].

Deliberative process:

1 The Other is regarded as not a threat but instead as a factor for expanding the horizon of freedom. Regarding the Other as such a factor enables meeting the 
imperative of developing an attitude of acceptance ('hospitality') when facing the unexpected (a 'foreigner'), and to regarding information from the unexpected as relevant to and valid for ourselves. Regarding such information in this way is through a dialogical process of understanding, recognizing, and possibly integrating or transforming the point of view of the Other: 'opening to the other involves recognizing that I myself should accept something against me... [6].

2 A problem is formulated in the way that it must be developed, through a deliberative process in dialogue, to reach an interpretation shared by the health care professional and the patient. Framing a problem in this way recognizes that ethics is not a matter of individual arguments or of ahistorical interpretation, but instead is a joint process involving the healthcare professional and the patient in learning. Such a process can happen only in dialogue, in a conversation with an intention of understanding, within a relationship of proximity that allows for comparisons of unexpected claims.

3 The result is not a theoretical knowledge of ethical principles and maxims or of abstract procedures, but rather a practical knowledge about what is best for a particular situation based on a casuistic-pragmatic perspective.

I conceptualized the model of relational ethics as one that embraces the meta-ethical principles of vulnerability, dignity, responsibility, and respect for autonomy as they are considered by many international declarations or conventions. It is an intermediate model that ensures prudent and reasonable decision-making (fig. 2).

The three structural elements and characteristics of relational ethics are (1) creating and maintaining a genuine, authentic, fair, respectful, and responsible relationship between the professional and the patient; (2) being aware that the mutual vulnerability between the professional and the patient has a moral value; (3) recognizing that their relationship will allow for personal development of each. This model integrates three key polarities: (1) ensure conditions of authenticity; (2) facilitate a process of cooperative mutuality; (3) promote opportunities for growth and development, taking into account the contingent and contextual relativity [7].

\section{Conditions of Authenticity}

The Other-sick person is temporarily unable to assume his or her own ontological autonomy for the attractive power of pain, other symptoms or suffering. It is the condition of authenticity [8] that provides for being surprised and for paying attention to live carnal, linguistic or cultural subjectivity that enables the professional, a subjective host, to be open to the infinity of the patient and to accept the patient's impatience imprisoned by suffering.

This condition of authenticity is manifested in a relational congruence in the questioning and dialogue, i.e. to a harmony between the health professional's inner attitude 




Fig. 2. Relational ethics model: theoretical framework.

and actual explicit behavior of the health professional. It is indispensable for openness to empathic accompaniment. Within the condition of authenticity, the health professional houses and at the same time approaches the patient's frame of reference and emotions, without dissolving or losing his or her own references. This condition creates a dynamics of mutual vulnerability resonance that becomes an indispensable 'existential and relational surveillance' that every health professional should internalize to minimize exploitation of the patient by the health professional and to ensure compliance.

If not viewed in this light, this relationship of 'asymmetric' vulnerability, in which health professionals and the patients have vulnerabilities, but patients are usually more vulnerable, can revive processes of projection, and denial, echoing in regressive and traumatic nuclei of health professionals. They risk being insensitive to and ignoring the real needs of others, or projecting their own suffering nuclei onto the patient. In so doing, they forget subconsciously or unconsciously the internal morality of the profession or the limits of ethical respect, or both, by reducing their attention and their care and solicitude, or by 'plastering' the normal life of the patient, or both. 
Identifying the emotional dimension in the relationship is crucial because it avoids different forms of manipulation arising from the following: inducing guilt and depreciation; sharing an alliance with the patient's pathological nuclei; delegating unfulfilled desires and unfinished tasks, or submitting the patient to one's own narcissistic gains. In another way, the relationship can deteriorate into deleterious therapeutic manipulations such as mechanical advice or suggestibility, thereby avoiding the deepening of the emotional and relational experience. It is basically through ethical work processes that the clinical work proceeds, and vice-versa.

So, what fundamentally characterizes the ethical stance of the health professional is to be inherently subject to the same determinants as what fundamentally characterizes the ethical stance of the patient. Only a constant questioning of oneself, which is sometimes difficult, can maintain respect for the frailty of physical, mental, and moral survival, but also that for the otherness.

\section{Process of Cooperative Mutuality}

The process of cooperative mutuality assumes that the principle of cooperation advocates that conversational postulates allow for resolving many problems of understanding the meanings of statements and declarations. The principle of cooperation promotes that communicators say things that are true and that are sufficiently informative, providing relevant information and avoiding unnecessary obscurities and ambiguities [9]. The principle holds that communicators do not say things for which there is lack of evidence.

The principle of cooperation also holds that communication should also be complemented by the principle of gentleness (tact, modesty, generosity). During the interactive process of communication, the principle of gentleness can be displayed by the countenance of the participants, who use courtesy as a sign of the absorption capacity of modes of housing being and doing, based on tenderness and sensitivity.

Stories and narratives of experiences expressed, restated, extended gradually, and made increasingly genuine through dialogue [10] will make explicit the implicit meaning of lived experiences [11]. Stories and narratives always contain a moral vision of the world. They present relevant information about who participates in those lived situations, about the relationships among and the responsibilities of participants. Stories and narratives promote the clarifying of problems and of moral responsibilities, asking for an interpretation. Ethical practice triggers the possibility of confrontation with patients' narratives, and in so doing, urges professionals to deal with situations that are often different from those that they know about, thus extending their perspectives and enabling internal change.

A disease defines and isolates a range of possibilities, such as obtaining secondary benefit, the aesthetics of suffering, masochism, and depression. This process of mutual coprocessing between professional and patient will allow this range of possibilities to 
extend the closed circle of the single mode condition of oneself (for which are given to understand the world and live in the real structure) to other existential possibilities.

What develops in a dual process involving patient and professional is exactly what develops in group processes of moral deliberation such as ethical reflection on the health care team in ethics committees. These group processes in which the participants' individual perspectives are extended by mutual confrontation of participants' views lead to the emergence of perspectives in which the individual views change to a common interpretation - a fusion of horizons in hermeneutics [6].

An intersubjective relationship requires both the removal of the primacy of the self as separate from the other. Therefore, intersubjectivity and mutual understanding have a tension and unstable dimension that is the real engine of any human relationship.

\section{Opportunities for Growth and Development}

The dimension of opportunities for growth and development arises from the argument that every human being has the potential to be transformed by interaction with others. Therefore, it is imperative that the opportunity for growth and development in a growing movement of interiority that relational involvement allows, stimulate the development of a new attitude to life, in which the individual is questioned more deeply about his or her values.

A disease can be understood as a disruption of an appropriate relationship with one self's integrity and unity. And it is from the creation of conditions of authenticity and from a process of cooperative mutuality that enlarging and transforming meanings of broken or distorted relationships may emerge as growth. It is the only condition for the patient to grow emotionally and ethically, and the same should happen to the health professional, as a sign that he established an ethically validated relationship.

Through the model of relational ethics, it is possible to evaluate positive and negative aspects of health, life events, and the context of bereavement reactions, and it is possible to check allostatic load and the role of interpersonal relationships in a process that facilitates self-management and shared decision making.

The health professional and patient jointly work on a discovery process that is collaborative, continuous, and flexibly revalued. This process allows for growth and development of both participants in respecting the complexity of the clinical situation and its organizational and environmental matrix [7].

\section{Process of Deliberation}

Relational ethics, which springs from perception of others' suffering and vulnerability, is aiming to build a response to a concern seeking correction of actions in clinical 
situations, searching the best for the patient, considering him as an end rather than a means (the Other as I), and taking into account the intention of the action.

The process of ethical decision is not limited to mere 'indifferent' compliance to the right principle (ignoring the singular circumstances) or to conduct that 'must be done' (reducing the uncertainty that is always present). It seeks the best possible understanding of all factors that influence the action. The process of ethical decision involves, through a relational ethics, listening to the patient's history and getting into a helical loop in which the professional and the patient together weigh values, beliefs, expectations, standards, and principles, with a focus on moral responsibility for each other in a life situation within a particular environmental context [12].

In clinical ethics there are no dogmas, no dilemmas, and no moral predicament. Instead, there are problems [13]. Decisions about solving these problems might not be fully rational, but they are more or less reasonable in the same way that values that give meaning to life might not be fully rational but are reasonable. Solutions might not be entirely certain, but they are likely to be certain in that a given problem might have multiple solutions. Decision-making is based on the principle that moral problems cannot always be solved, and that solutions to moral problems do not always have only one answer that is always the same. The deliberative process acts as a mode of knowledge and as a process that is not split of evolution and of changing an existing viewpoint from which it might have departed.

Instead, the deliberative process is one that emerges enriched in knowledge, understanding, and recognition of others.

The deliberative process is a procedure that seeks to enrich the analysis of a clinical situation to increase prudence in decision-making. It aims to improve the quality of clinical decision making by proper managing values. It optimizes solving conflicts of values. Its overall objective is to learn how to deal with moral uncertainty with the same sufficiency with which health professionals deal with clinical uncertainty. The deliberation process specifically seeks to identify the conflicting values, to make prudent and reasonable decisions that are not necessarily uniform, unanimous, or consensual, and to adopt optimal decisions that are the best possible and most mature. This process is not only from the standpoint of clinical facts but also from the standpoint of values.

The process of deliberation undergoes three steps: (1) analysis of clinical facts; (2) analysis of conflict of values, and (3) deliberation about courses of action.

In the first step, health professionals analyze the clinical facts relevant to the outcome and then identify the arising of ethical problems, selecting those most pertinent to the situation.

In the second step, health professionals analyze relevant values held by themselves and by others, who include patients, family members, caregivers, other heath professionals, organizations, and the community. In analyzing relevant values, health professionals understand that an ethical problem is always a problem of conflicts of values that must be identified while taking into account that the duty of 
a health professional is the achievement of values that are not fully rational but reasonable.

In a third step, health professionals decide on the various possible courses of action to achieve the best possible solution. After evaluating the courses of action and having checked them against their consequences, a health professional elects the optimal course of action that will be defended from the values underlying it. The process is completed by taking into account three types of proofs of consistency: legality, publicity, and temporality [12].

The detailed procedures for ethics deliberation are as follows:

1 Identifying medical problems through a biopsychosocial perspective

2 Identifying the relevant data of ethical problems

3 Identifying values involved

4 Identifying possible courses of action

5 Comparing courses of action with the ethical principles involved

6 Appreciating the consequences of actions, which includes justifying possible exceptions

7 Contrasting the course of action with the canon

8 Implementing all actions taken through relational ethics procedures.

\section{Major Ethical Problems in Psychosomatic Medicine}

Psychosomatic medicine professionals are largely engaged in clinical problems that often are ethics consultations [14] in which they are called to play the role of experts in clinical ethics [15]. Examples of main ethical problems in this regard are capacity, informed consent, and confidentiality.

\section{Capacity}

Capacity is an ability to perform a given task. It refers to mental abilities, cognitive or behavioral requirements, to perform an action or a task with specific legal implications or to set a civilian role [16]. Capacity is specific to a given situation and may vary over time. In relation to specific tasks, capacity may vary from one context to another: Although someone might (or might not) have the capacity to decide about financial issues, that person might (or might not) have the capacity to decide about health issues, such as agreeing to undergo recommended diagnostic or therapeutic procedures or to participate in research.

Health professionals in the field of psychosomatic medicine are often requested to assess patients' capacities in the context of ethical decision-making in various situations. These situations include procedural matters, such as informed consent, accepting undergoing diagnostic or treatment procedures or refusing to undergo them or 
hospital discharge. Or the situations can relate to a patient's condition, such as illness denial, type A behavior, irritable mood, anorexia, demoralization, disease phobia, or persistent somatization.

In the context of various difficulties and the possibility of bias, health professionals need to have clarified some levels, criteria, and basic parameters for assessing the various components of capacity: communication of a decision, understanding the information provided, assessing the options provided, and a process of rational decision $[17,18]$, and doing so the three components of relational ethics are particularly pertinent.

The ability to decide is the ability to appreciate the significance of the decision in the context of one's personal history and personal values [19]. This ability is deeply shaped by the history of each individual and his or her neurocognitive capacity for insight. The focus is not the rationality of a decision. It is on the rationality of the thought process leading to a decision.

Several clinical factors can contribute to determining the capacity to consent to recommended diagnostic or treatment procedures, and they need to be taken into consideration: quantity and quality of information available, stability of patient's mental status, and psychopathological and psychodynamic factors.

\section{Quantity and Quality of Information Available to the Patient}

The quantity and quality of information available to a patient should be evaluated because a patient who has been uninformed or misinformed may seem to lack capacity. Limiting the provision of information directly to the patient as the sole benchmark for assessing capacity can lead to errors because the patient might not disclose or even deliberately hide information and symptoms. It is essential to compare and contrast patient-provided information with that from informants.

\section{Stability of a Patient's Mental Status}

A patient's mental status is sometimes not very stable, varying with clinical course and requiring additional evaluations. Incompetence can be temporary when level of consciousness fluctuates with or depends on disease course. Sometimes the cause of incapacity, such as pain, can be treated or relieved. Stimulants can restore a depressed patient's perspective. Neuroleptics may alleviate a patient's confusion [20]. In such situations, a professional can wait before carrying out further assessments of capacity. Even a psychotic patient may have reasons to refuse treatment.

\section{Psychopathological and Psychodynamic Factors}

The main psychiatric aspects usually attributed to promoting ethical difficulties are as follows: diagnosable psychopathology in any of the stakeholders of an ethical conflict; personality styles and psychodynamic issues; family dysfunction; interpersonal conflicts among patient, family, and health professionals; conflict within or lack of cooperation among members of the healthcare team, and countertransference reactions 
of health professionals or disproportionate involvement in, or rejection by the health team toward patients, families and other staff.

In medical ethics, most problems with practical clinical implications require resolution of conflicts between patients, physicians, and nurses. Naturally, often unconscious intrapersonal conflicts are behind these problems [21]. Issues of ethical conflicts are apparently caused by faulty emotional or ethical difficulties and problems that mask patients' emotional problems [22]. The psychiatrist's recognizing these emotional or psychiatric factors is essential to understanding and resolving the ethical conflict.

Some treatments and procedures arouse fear and anxiety in some patients, sometimes leading patients to control these emotions by regressive movements, including psychotic ones. For example, a patient with ulcerative colitis with an indication for colostomy may fear being abandoned by his or her immediate family after the operation. This fear can lead the patient to defend himself or herself in an ever more regressive and even psychotic denial of disease and distortion of reality. Some traits or diseases may also skew the assessment of capacity such as alexithymia traits, psychosis, or cognitive distortions in depression [23].

One of the primary responsibilities of psychiatrists in dealing with ethical conflicts in medical treatment is not only to assess the patient's capacity, but also to correct the diminished capacity [24], and also to treat reversible mental disorders whose relief might enable the entire process of informed consent. The following situations can trigger different types of psychiatric interventions: dealing with individual psychological problems; evaluating the ability to decide; restoring, enhancing, stabilizing decisionmaking capacity; improving the dynamics of dysfunctional families; diagnosing and reducing interface problems within the health care team and among the health care team, the patient, and family; involving other caregivers if necessary; recognizing and minimizing cultural or religious inconsistencies, and evaluating and optimizing the contribution of the society or community to the patient's well-being.

Incapacity cannot be assumed simply because patient is under psychiatric treatment, hospitalized, or institutionalized. A disability or a mental disorder by itself does not render a person incapable in every area of functioning. Therefore, patients should be rigorously evaluated to detect specific functional impairments that render them unable to take a particular type of decision or to perform certain type of tasks.

\section{Informed Consent}

From the perspective of relational ethics, informed consent should be regarded not as a moment but as a process, a relational opportunity, and a moment of personal growth and development. This process prioritizes the aspects of communication, interaction, and relationship, referring to the formal consent into the background.

Informed consent is an act, an authorization of an autonomous person [25] allowing a health professional to perform a diagnostic or therapeutic procedure that has 
been explained. The three conditions to be met in informed consent are capacity, voluntariness, and consistency.

For the condition of capacity to be met, the patient must have the mental capacity to, in view of concrete situations that he or she is facing, perform functions of analyzing, understanding, and deciding.

The condition of voluntariness (willingness) has to do with free choice. The decision to choose from several options should be voluntary, and this decision must be checked to find out if it has been made under coercion or threat. Coercion can occur, sometimes defended on the grounds that there is valid justification that a particular choice is in the patient's best interest, so that the application of coercion is in the patient's best interest [26].

We must check also if a decision is made in response to intense fears. In such an instance, it is necessary to evaluate the proportion between natural and understandable fears and irrational fears. The patient may also be paranoid or have other psychological problems, such as major depression with its feelings of profound helplessness, worthlessness, and demoralization. Paranoia and depression seriously affect free choice. The presence of depression may influence how the patient tolerates uncomfortable symptoms and maintains hope in relation to the risk and benefit of treatment, but it cannot make him incapable of making decisions for himself or herself.

The condition of consistency has to do with a patient's ability to make a decision and adhere to it. Hesitation or indecision is considered as the patient's not being sure. The health care professional should evaluate the consistency or inconsistency in a patient's decisions if the patient changes his or her mind with new information. In regard to the health care professional's fiduciary responsibility, he or she should serve as the trusted guardian because of the profound consequences of some medical decisions and because the patient's reasoning and reasoning and decision-making processes can be affected by fear and distorted beliefs, somatic illnesses, and medications.

\section{Confidentiality}

Confidentiality is one of the most solid foundations of the trust between health professional and patient. It is essential for achieving the goals of a clinical relationship. The patient has the right to confidentiality, and the health professional has a duty, the professional obligation, of keeping secret the information given by the patient. Simultaneously, maintaining confidentiality is also a way to build feelings of trust and safety in working with the medical profession.

It is very difficult to maintain confidentiality in the hospital or inpatient psychosomatic settings in which many professionals are seeing the same patient [27]. In this context, there may be a tendency not to protect the patient's confidentiality with the same zeal as that within outpatient settings. Considering the complexity and severity of clinical situations, it is natural that the anxiety and high stress may interfere 
with professional judgment. Moreover, the need for involvement of family and for other medical and nonmedical staff in a hospital means that a lot of information is collected from various sources. In such situations, confidentiality cannot be assured zealously.

The psychiatrist working with the medical team has a duty to provide relevant information to various team members to enable them to develop more accurate assessments and to plan appropriate treatments. Disclosure of confidential information that contextualizes some of the patients' behaviors and attitudes may be particularly important to avoid misunderstandings and miscommunications. Such disclosure may facilitate a cooperative team relationship that will benefit the patient.

But on the other hand, it is essential to retain a degree of intimacy [28] that does not need to be shared with the entire team. Some subjects covered by the patient cannot be disclosed because they are not sufficiently relevant to the work of other medical professionals and to nursing decision-making. The psychiatrist needs to balance between the need for information for the medical team to assess the patient's condition and the need to maintain patient's privacy. The psychiatrist must be very scrupulous in filtering information that should be held confidential from information needed to help the team.

\section{Conclusions}

In the context of a health system with a tendency to fragmentation, psychosomatic medicine approaches often complex cases for which it is necessary to use new clinical assessment methods that lead directly to integrated therapeutic interventions.

These complex cases, demanding multisystem evaluations, multidisciplinary approaches, and multiple therapies require the development of methods of inquiry and of appropriate ethical deliberation that allow early identification of problems and determination of optimal courses of action.

The method of clinically centered relational ethics, involving a partnership with science and philosophy and promoting a deliberative process, is particularly suitable to comprehensively address common ethical problems in the practice of and research in psychosomatic medicine.

Relational ethics ensures conditions of authenticity to facilitate a process of cooperative mutuality to promote opportunities for growth and development of the health professional and patient partners. In doing so, relational ethics takes into account contingent and contextual relativity. These are the basic premises that allow for ethical reflection by integrating the main theoretical principles and perspectives not only from the standpoint of clinical facts but also from that of values to make prudential and reasonable decisions [7]. 


\section{References}

1 Barbosa A: Ética relacional; in Barbosa A, Neto I (eds): Manual de Cuidados Paliativos. Lisboa, Núcleo de Cuidados Paliativos/Centro de Bioética/ Faculdade de Medicina de Lisboa, 2006, pp 439452.

2 American College of Physicians: Ethics Manual. Ann Intern Med 1992;117:947-960.

3 Dickenson D, Fulford KWM (eds): In Two Minds: A Casebook of Psychiatric Ethics. Oxford, Oxford University Press, 2000.

4 Steinkam NL: European debates on ethical case deliberation. Med Health Care Philos 2003;6:225226.

5 Birley J: Psychiatric ethics: an international open society; in Dickenson D, Fulford KWM (eds): In Two Minds: A Casebook of Psychiatric Ethics. Oxford, Oxford University Press, 2000, pp 329-335.

6 Gadamer HG: O mistério da saúde: $\mathrm{O}$ cuidado da saúde e a arte da medicina. Lisboa, Edições 70, 2009.

7 Barbosa A: Ética relacional; in Barbosa A, Neto I (eds): Manual de Cuidados Paliativos, ed. 3, revised. Lisboa, Núcleo de Cuidados Paliativos/Centro de Bioética/Faculdade de Medicina de Lisboa, 2010, pp 661-691.

8 Arnason V: Towards authentic conversations authenticity in the patient professional relationship. Theor Med 1994;153:227-242.

9 Habermas J: Morale et communication: conscience morale et activité communicationnelle. Paris, Cerf, 1986.

10 MacIntyre A: After the Virtue. London, Duckworth, 1981.

11 Ricoeur P: Soi-même comme un autre. Paris, Seuil, 1990.

12 Barbosa A: Processo de deliberação ética; in Barbosa A, Neto I (eds): Manual de Cuidados Paliativos, ed. 3, revised. Lisboa, Núcleo de Cuidados Paliativos/ Centro de Bioética/Faculdade de Medicina de Lisboa, 2010, pp 693-721.

13 Gracia D: Moral deliberation: the role of methodologies in clinical ethics. Ethical case deliberation and decision making. Med Health Care Philos 2001;4: 223-232.
14 Steinberg MD: Psychiatry and bioethics an exploration of the relationship. Psychosomatic 1997;38:313320.

5 Binder RL: Liability for the psychiatrist expert witness. Am J Psychiatry 2002;159:1819-1825.

16 Pomerantz AS, Nesnera A: Informed consent competency and the illusion of rationality. Gen Hosp Psychiatry 1991;132:138-142.

17 Appelbaum PS, Grisso T: Assessment patient's capacities to consent to treatment. N Eng J Med 1988;319:1635-1638.

18 Appelbaum PS, Grisso T: Capacities of hospitalized medically ill patients to consent to treatment. Psychosomatics 1997;38:119-125.

19 Welie SP: Criteria for patient decision making in competence: a review of and commentary on some empirical approaches. Med Health Care Philos 2001;4:139-151.

20 Adamis D, Martin FC, Treloar A, Macdonald AJ: Capacity consent and selection bias in a study of delirium. J Med Ethics 2005;31:137-143.

21 Kornfeld DS: Clinical ethics: an important role for the consultation liaison psychiatrist. Acad Psychosom Med 1997;384:307-308.

22 Choen LM, Fischel S, Germain M, Woods A, Braden GL, McCue J: Ambivalence and dialysis discontinuation. Gen Hosp Psychiatry 1996;18:431-435.

23 Kramer PD: The valorization of sadness. Hastings Center Report 2000;30:213-218.

24 Cassel EJ, Leon AC, Kaufman StG: Preliminary evidence of impaired thinking in sick patients. Ann Intern Med 2001;134:1120-1123.

25 DeGrazia D: Autonomous action and autonomy subverting psychiatric conditions. J Med Philos 1994;19:279-297.

26 Liegeois A, Eneman M: Ethics of deliberation consent and coercion in psychiatry. J Med Ethics 2008; 34:73-76.

7 Green SA: The ethical limits of confidentiality in the therapeutic relationship. Gen Hosp Psychiatry 1995; 17:80-84.

28 Lipkin R: Intimacy and confidentiality in psychotherapeutic relationships. Theor Med 1989;10:311330 .

António Barbosa

Centro de Bioética/Faculdade de Medicina de Lisboa

Av. Prof. Egas Moniz

PT-1649-028 Lisboa (Portugal)

Tel. +35 1217985182, E-Mail abarbosa@netcabo.pt 